\title{
Chumbo em solos às margens de estradas na região metropolitana de Curitiba
}

\section{Lead contents in soils around highways sides in Curitiba's metropolitan region}

\author{
Caroline Zanello Broska ${ }^{[a]}$, Carolina Maris de Andrade ${ }^{[a]}$, \\ Sonia Zanello ${ }^{[b]}$, Vander de Freitas Melo ${ }^{[c]}$ \\ [a] Tecnóloga em Química Ambiental pela Universidade Tecnológica Federal do Paraná (UTFPR), Curitiba, PR - Brasil, e-mail: \\ cbroska@essencis.com.br \\ [b] Química, Mestre em Ciência do Solo, professora do Departamento de Química e Biologia da Universidade Tecnológica Federal \\ do Paraná (UTFPR), Curitiba, PR - Brasil, e-mail: zanello@utfpr.edu.br \\ [c] Engenheiro agrônomo, Doutor em Solos e Nutrição de Plantas, professor do Departamento de Solos e Engenharia Agrícola da \\ Universidade Federal do Paraná (UFPR), Curitiba, PR - Brasil, e-mail: vanderfm@ufpr.br
}

\section{Resumo}

Tendo em vista que o tráfego rodoviário pode ser fonte potencial de metais pesados que se depositam nos solos ao longo das estradas, objetivou-se no presente trabalho avaliar a concentração de chumbo $(\mathrm{Pb})$ às margens da BR 116 e BR 277, em trechos em que estas rodovias passam por Curitiba e São José dos Pinhais, respectivamente. Amostras de solos foram tomadas em sete pontos próximos às rodovias e em dois pontos distantes $4 \mathrm{~km}$ delas, para servirem de referência quanto aos níveis naturais de $\mathrm{Pb}$ nos solos advindos do material de origem. Realizou-se a análise textural e química dos solos. Os teores totais e trocáveis de $\mathrm{Pb}$ foram determinados por espectroscopia de absorção atômica. Os teores mais elevados de $\mathrm{Pb}$ foram observados na amostra $\mathrm{P}_{3}\left(215,1 \mathrm{mg} \mathrm{kg}^{-1}\right)$, que recebe grande volume de água de escorrimento superficial da rodovia em eventos de precipitação, e nas amostras do ponto $\mathrm{P}_{1}$ (horizonte $\mathrm{A}-41,7 \mathrm{mg} \mathrm{kg}^{-1}$ e horizonte $\mathrm{Cg}-44,1 \mathrm{mg} \mathrm{kg}^{-1}$ ), ao lado do posto da polícia rodoviária na $\mathrm{BR}$ 277. O incremento de $\mathrm{Pb}$ nas amostras do $\mathrm{P}_{1}$ em relação ao solo de referência foi atribuído à deposição direta do metal particulado presente na fumaça liberada pelos veículos.

Palavras-chave: $\mathrm{Pb}$ particulado. Contaminação do solo. Tráfego rodoviário.

\begin{abstract}
Bearing in mind that the road traffic may be a potential source of heavy metals which deposit along the highways, the present work has as objective to evaluate the lead $(\mathrm{Pb})$ concentration on the side of BR 116 and BR 277 in Curitiba, PR. Soil samples were collected in the seven sites besides the highways and in two control sites at a distance of $4 \mathrm{~km}$ distance from the highway. Textural and chemical analyses were made in soil samples. The Pb total and exchangeable contents were determined by atomic absorption
\end{abstract}


spectrometry. The highest $\mathrm{Pb}$ contents were observed in sample $P_{3}\left(215.1 \mathrm{mg} \mathrm{Kg}^{-1}\right)$ which receives surface dripping water from the road during precipitation events, and in samples $P_{1}$ (A horizon - 41.7 $m g \mathrm{~kg}^{-1}$ and $C$ horizon - $44.1 \mathrm{mg} \mathrm{kg}^{-1}$ ), next to traffic police post. The Pb contents increasing in the $P_{1}$ soil samples in relation of soil control was attributed to direct deposition of the particulate metal from vehicles.

Keywords: Particulate Pb. Soil contamination. Road traffic.

\section{Introdução}

A principal origem da contaminação do ambiente por chumbo $(\mathrm{Pb})$ são as emissões atmosféricas provenientes do tráfego rodoviário e de fundições de metais, fábricas de baterias e indústrias químicas, principalmente quando não utilizam filtros de ar durante o processo industrial (LANDRIGAN, 2002; VANZ; MIRLEAN; BAISCH, 2003; TURER; MAYNARD, 2003).

Embora as emissões de $\mathrm{Pb}$ tenham diminuído drasticamente com a eliminação progressiva de $\mathrm{Pb}$ tetraetila como aditivo de combustível, este metal depositado ao longo das rodovias continua a ser motivo de preocupação, graças à sua não biodegradabilidade e acúmulo na superfície do solo em longo prazo e à toxicidade (SUTHERLAND et al., 2000; LANDRIGAN, 2002; LI, 2006). Atualmente, o principal uso do Pb é na fabricação de baterias de chumbo-ácido utilizadas em veículos automotivos, porém, este metal também está presente em outros componentes dos veículos, como pastilhas de freio e pneus (DOLAN et al., 2006).

$\mathrm{O}$ aumento da concentração de $\mathrm{Pb}$ nos solos está altamente correlacionado com o inverso da distância às rodovias. Das emissões de chumbo lançado por automóveis, $20 \%$ a $60 \%$ são depositados próximo à fonte geradora, o que é observado pelos altos teores deste metal nos vegetais e nos solos ao longo das rodovias de tráfego intenso (WHO, 1995; VANZ et al., 2003; TURER; MAYNARD, 2003). O índice de contaminação também é influenciado pela velocidade dos ventos e precipitação (VIARD et al., 2004).

$\mathrm{O} \mathrm{Pb}$ originado do tráfego rodoviário pode chegar aos solos e vegetais após um evento de precipitação, por meio das águas de escorrimento superficial e/ou da deposição atmosférica (TURER; MAYNARD, 2003). Dependendo da composição e condições de saturação do solo, esse metal pode ser transferido para a cadeia alimentar, chegar às águas subterrâneas ou ficar retido no solo.

Os principais parâmetros que influenciam a disponibilidade de metais pesados no solo são: textura, $\mathrm{pH}$, tipo de mineral da fração argila (minerais silicatados e óxidos de Fe e de Al), teor de matéria orgânica e tipo e teor de cátions e ânions no solo (WENG et al., 2002; ANDRADE et al., 2003; WU et al., 2003).

No Brasil, há poucos estudos referentes à contaminação dos solos por metais pesados às margens de estradas, decorrentes da queima de combustíveis fósseis e desgaste dos veículos automotivos. Portanto, o objetivo deste trabalho foi avaliar os teores totais e trocáveis de $\mathrm{Pb}$ nos solos às margens das rodovias BR-116 e BR-277, para averiguar se ocorreu incremento desse metal em decorrência do tráfego de veículos.

\section{Material e métodos}

As amostras de solos foram coletadas no entorno das BR 116 e 277, nos trechos em que as rodovias passam por Curitiba e São José dos Pinhais, PR, respectivamente, onde o tráfego de veículos é intenso. No ponto $\mathrm{P}_{1}$ as amostras foram tomadas nas profundidades de 0 a $25 \mathrm{~cm}$ (horizonte A) e de 50 a $70 \mathrm{~cm}$ (horizonte $\mathrm{Cg}$ ) (Tabela 1). Esse ponto foi o único tomado de barranco às margens da rodovia, onde havia a exposição de mais de um horizonte pedogenético à fumaça emitida pelos carros, o que justificou sua amostragem em duas profundidades. Nos demais pontos $\left(\mathrm{P}_{2}, \mathrm{P}_{3}, \mathrm{P}_{4}, \mathrm{P}_{5}, \mathrm{P}_{6}\right.$ e $\left.\mathrm{P}_{7}\right)$, as mostras de solo foram coletadas apenas na camada superficial (0 a $5 \mathrm{~cm}$ ), pois não havia exposição lateral dos horizontes (pontos na mesma cota da superfície do pavimento). $\mathrm{O} \mathrm{Pb}$, ao se infiltrar no solo com a água das chuvas, acumula-se preferencialmente no topo do perfil, pela alta associação do poluente com os componentes da fração húmica (GOMES et al., 1997; AMARAL SOBRINHO et al., 1998; ANDRÉ et al., 2003). 
Os pontos $\mathrm{R}_{1}$ e $\mathrm{R}_{2}$ (Gleissolo e Cambissolo, respectivamente) foram tomados como referência para estabelecer os teores naturais de $\mathrm{Pb}$ (oriundos do material de origem), pois os locais estavam supostamente livres de influência antrópica. As amostras do ponto $\mathrm{R}_{2}$ (referências para $\mathrm{P}_{3}, \mathrm{P}_{4}$ e $\mathrm{P}_{5}$ - Tabela 1) foram coletadas nos horizontes A, B e C, com o propósito de acompanhar os teores naturais de $\mathrm{Pb}$ ao longo do perfil, pois os pontos $\mathrm{P}_{3}, \mathrm{P}_{4}$ e $\mathrm{P}_{5}$ (coleta de 0 a $5 \mathrm{~cm}$ ) apresentavam-se decapeados pelos trabalhos de engenharia nas rodovias. Para os teores de referência de Pb para os pontos P6 e P7 (BR 116 - argilito da Formação Guabirotuba), tomaram-se os dados do trabalho de Pires et al. (2007).

Tabela 1 - Material de origem, classificação dos $\operatorname{solos}^{(1)}$ e detalhes dos ambientes de amostragem

\begin{tabular}{|c|c|c|c|c|}
\hline $\begin{array}{l}\text { Ponto/ horizonte } \\
\text { e profundidade de } \\
\text { coleta }(\mathrm{cm})^{(2)}\end{array}$ & $\begin{array}{l}\text { Material de } \\
\text { origem }\end{array}$ & Classe & $\begin{array}{l}\text { ZONAS } \\
\text { (UTM) }\end{array}$ & Descrição \\
\hline $\begin{array}{l}\mathrm{P}_{1} / \mathrm{A}(0 \text { a } 25 \mathrm{~cm}) \\
\mathrm{P}_{1} / \mathrm{Cg}(50 \text { a } 70 \mathrm{~cm})\end{array}$ & $\begin{array}{l}\text { Sedimentos } \\
\text { Argilo-siltosos }\end{array}$ & Gleissolo & $\begin{array}{l}0695432 \mathrm{~W} \\
7173402 \mathrm{~S}\end{array}$ & $\begin{array}{l}\text { BR 277: ao lado do Posto da Polícia Rodoviária Federal, } \\
\text { sentido Curitiba-Paranaguá. Apresenta hidromorfismo e } \\
\text { nível freático elevado. Não recebe águas de escorrimento } \\
\text { superficial da rodovia; apenas a possibilidade de } \\
\text { incremento de Pb particulado, proveniente da fumaça dos } \\
\text { escapamentos dos veículos. Altitude } 921,8 \mathrm{~m} \text {. }\end{array}$ \\
\hline $\mathrm{P}_{2}^{(3)}(0$ a $5 \mathrm{~cm})$ & $\begin{array}{l}\text { Sedimentos } \\
\text { Argilo-siltosos }\end{array}$ & Gleissolo & $\begin{array}{l}0690050 \mathrm{~W} \\
7176193 \mathrm{~S}\end{array}$ & $\begin{array}{l}\text { BR 277: ao lado da pista oposta à montadora da Renault } \\
\text { (São José dos Pinhais). Apresenta hidromorfismo e } \\
\text { nível freático elevado. Recebe águas de escorrimento } \\
\text { superficial da rodovia. Altitude } 905,0 \mathrm{~m} \text {. }\end{array}$ \\
\hline $\mathrm{P}_{3}^{(3)}(0 \mathrm{a} 5 \mathrm{~cm})$ & Granito & Cambissolo & $\begin{array}{l}0698305 \mathrm{~W} \\
7171951 \mathrm{~S}\end{array}$ & $\begin{array}{l}\text { BR 277: ao lado da pista, após o pedágio no sentido } \\
\text { Curitiba-Paranaguá. Recebe águas de escorrimento } \\
\text { superficial da rodovia. Altitude } 962,5 \mathrm{~m} \text {. }\end{array}$ \\
\hline $\mathrm{P}_{4}^{(3)}(0$ a $5 \mathrm{~cm})$ & Granito & Cambissolo & $\begin{array}{l}0698326 \mathrm{~W} \\
7171933 \mathrm{~S}\end{array}$ & $\begin{array}{l}\text { BR 277: ao lado da pista, após o pedágio no sentido } \\
\text { Curitiba-Paranaguá (próximo ao } \mathrm{P}_{3} \text { ). Recebe águas de } \\
\text { escorrimento superficial da rodovia. Altitude } 964,6 \mathrm{~m} \text {. }\end{array}$ \\
\hline $\mathrm{P}_{5}^{(3)}(0$ a $5 \mathrm{~cm})$ & Granito & Cambissolo & $\begin{array}{l}0700953 \mathrm{~W} \\
7170626 \mathrm{~S}\end{array}$ & $\begin{array}{l}\text { BR 277: ao lado da pista, após o pedágio no sentido } \\
\text { Curitiba-Paranaguá (ponto mais próximo ao pedágio } \\
\text { em relação ao } \mathrm{P}_{3} \text { e } \mathrm{P}_{4} \text { ). Recebe águas de escorrimento } \\
\text { superficial da rodovia. Altitude } 912,0 \mathrm{~m} \text {. }\end{array}$ \\
\hline $\mathrm{P}_{6}^{(3)}(0 \mathrm{a} 5 \mathrm{~cm})$ & Argilito ${ }^{(4)}$ & Cambissolo & $\begin{array}{l}0677420 \mathrm{~W} \\
7184223 \mathrm{~S}\end{array}$ & $\begin{array}{l}\text { BR 116: ao lado da pista, próximo a passarela da UFPR. } \\
\text { Recebe águas de escorrimento superficial da rodovia. } \\
\text { Altitude: } 911,5 \mathrm{~m} .\end{array}$ \\
\hline $\mathrm{P}_{7}^{(3)}(0$ a $5 \mathrm{~cm})$ & Argilito ${ }^{(4)}$ & Cambissolo & $\begin{array}{l}0676115 \mathrm{~W} \\
7183414 \mathrm{~S}\end{array}$ & $\begin{array}{l}\text { BR 116: ao lado da pista, às margens do Rio Belém. } \\
\text { Recebe águas de escorrimento superficial da rodovia. } \\
\text { Localiza-se em cota mais baixa, mas no mesmo } \\
\text { seguimento de reta do } \mathrm{P}_{7} \text {. Altitude: } 894,5 \mathrm{~m} \text {. }\end{array}$ \\
\hline $\begin{array}{l}\mathrm{R}_{1} / \mathrm{A}(0 \text { a } 25 \mathrm{~cm}) \\
\mathrm{R}_{1} / \mathrm{Cg}(50 \text { a } 70 \mathrm{~cm})\end{array}$ & $\begin{array}{l}\text { Sedimentos } \\
\text { Argilo-siltosos }\end{array}$ & Gleissolo & $\begin{array}{l}0695197 \mathrm{~W} \\
7172765 \mathrm{~S}\end{array}$ & $\begin{array}{l}\text { Área sob vegetação natural. Local distante } 4 \mathrm{~km} \text { da rodovia } \\
\text { BR } 277 \text {, transversalmente ao Posto da Polícia Rodoviária } \\
\text { Federal }\left(\mathrm{P}_{1}\right) \text {. Altitude: } 940,0 \mathrm{~m} \text {. }\end{array}$ \\
\hline $\begin{array}{l}\mathrm{R}_{2} / \mathrm{A}(0 \text { a } 20 \mathrm{~cm}) \\
\mathrm{R}_{2} / \mathrm{Bi}(20 \text { a } 40 \mathrm{~cm}) \\
\mathrm{R}_{2} / \mathrm{C}(40 \text { a } 60 \mathrm{~cm})\end{array}$ & Granito & Cambissolo & $\begin{array}{l}0701221 \mathrm{~W} \\
7169941 \mathrm{~S}\end{array}$ & $\begin{array}{l}\text { Área sob vegetação natural. Local distante } 4 \mathrm{~km} \text { da rodovia } \\
\text { BR } 277 \text {, transversalmente ao pedágio Curitiba-Paranaguá } \\
\left(\mathrm{P}_{3}, \mathrm{P}_{4}, \mathrm{P}_{5} \text { e } \mathrm{P}_{6}\right) \text {. Altitude: } 958,8 \mathrm{~m} \text {. }\end{array}$ \\
\hline
\end{tabular}

Legenda: ${ }^{(1)}$ EMBRAPA (2006). (2) $\mathrm{R}_{1} / \mathrm{A}$ e $\mathrm{R}_{1} \mathrm{C}$ - amostras referências para os pontos $\mathrm{P}_{1}$ e $\mathrm{P}_{2} ; \mathrm{R}_{2} / \mathrm{A}, \mathrm{R}_{2} \mathrm{~B}$ e $\mathrm{R}_{2} \mathrm{C}$ - amostras referências para os pontos $\mathrm{P}_{3}, \mathrm{P}_{4}$ e $\mathrm{P}_{5}$. Para os teores de referência de $\mathrm{Pb}$ (teores naturais oriundos do material de origem) para os pontos $\mathrm{P}_{6}$ e $\mathrm{P}_{7}$ (BR 116 - argilito da Formação Guabirotuba) tomou-se o trabalho de Pires et al. (2007). ${ }^{(3)}$ Solo decapeado, sendo a amostra coletada no topo da camada superficial (0 a 5 cm). ${ }^{(4)}$ Argilito da Formação Guabirotuba. 
Após a coleta, as amostras foram secas ao ar e passadas em peneira com $2 \mathrm{~mm}$ de abertura, para obtenção da terra fina seca ao ar (TFSA). Em seguida, realizou-se a análise granulométrica, utilizando-se o método da pipeta e as análises químicas de rotina, de acordo com a Embrapa (1999).

Para a obtenção dos teores totais de $\mathrm{Pb}$, foi realizada a digestão ácida das amostras de solo (LIM; JACKSON, 1986, com algumas adaptações). Optou-se, para evitar perdas por volatilização e contaminação, utilizar cadinhos de teflon com tampas em rosca. Pesaram-se 0,2 g de TFSA previamente seca em estufa a $60^{\circ} \mathrm{C}$ por $48 \mathrm{~h}$. Em seguida, foram adicionados $5 \mathrm{~mL}$ de $\mathrm{HF}, 1 \mathrm{~mL}$ de $\mathrm{HNO}_{3}$ e $1 \mathrm{~mL}$ de $\mathrm{HClO}_{4}$ concentrados, e os cadinhos de teflon foram levados ao banho de areia pré-aquecido a $230^{\circ} \mathrm{C}$, no qual permaneceram por 90 minutos. Após o resfriamento dos cadinhos, repetiu-se o processo sem adicionar $\mathrm{HClO}_{4}$. Ao fim da digestão e resfriamento dos cadinhos, o líquido foi transferido para recipientes plásticos, utilizando-se água deionizada para lavar o cadinho de teflon, e a massa de extrato foi aferida para $15 \mathrm{~g}$. $\mathrm{O}$ volume foi determinado com base na densidade do extrato. A determinação dos teores de $\mathrm{Pb}$ foi por espectrometria de absorção atômica (EAA).

Para a determinação dos teores trocáveis de $\mathrm{Pb}$ foram agitados $2 \mathrm{~g}$ de TFSA, previamente seca em estufa a $60^{\circ} \mathrm{C}$ por $48 \mathrm{~h}$, com $60 \mathrm{~mL}$ de solução de $\mathrm{MgCl}_{2} 0,5 \mathrm{~mol} \mathrm{~L}^{-1}$ por $2 \mathrm{~h}$, segundo o método proposto por Norrström e Jacks (1998). A suspensão foi filtrada e a determinação dos teores de Pb foi conforme descrito anteriormente.

Análises de correlações simples (Pearson) foram executadas entre características físicas e químicas dos solos com os teores de totais e trocáveis de $\mathrm{Pb}$, utilizando-se o Programa Assistat ${ }^{\circledR}$, desenvolvido no Centro de Tecnologia e Recursos Naturais da Universidade Federal de Campina Grande, PB.

\section{Resultados}

$\mathrm{O}$ teor de argila dos solos coletados às margens das rodovias foram superiores a $200 \mathrm{~g} \mathrm{~kg}^{-1}$, com exceção das amostras $\mathrm{P}_{3}$ e $\mathrm{P}_{5}$ (Tabela 2). Outro fator que favorece a retenção de metais pesados no solo, o que reduz a contaminação dos aquíferos (KABATA-PENDIAS; PENDIAS, 2001), é o alto valor de capacidade de troca de cátions (CTC total) das amostras $\mathrm{P}_{1}$ a $\mathrm{P}_{7}$. A matéria orgânica foi importante para aumentar a CTC dos solos (coeficiente de correlação, r, entre carbono orgânico, CO, e CTC total $=0,88 ; \mathrm{p}<0,004)$. Os teores de $\mathrm{CO}$ das amostras tomadas às margens das rodovias variaram de 23,2 a $105,0 \mathrm{~g} \mathrm{~kg}^{-1}$.

A reação ácida dos solos ( $\mathrm{pH}$ baixo) foi coerente com os altos teores de $\mathrm{Al}^{3+}$ trocável e de acidez potencial não trocável $(\mathrm{H})$, responsável pelo bloqueio de grande parte das cargas negativas dependentes de $\mathrm{pH}$, principalmente da fração húmica ( $\mathrm{r}$ entre $\mathrm{CO}$ e $\mathrm{H}=0,77 ; \mathrm{p}<0,02)$. Os maiores valores de $\mathrm{pH}$ das amostras $\mathrm{P}_{5}$ e $\mathrm{P}_{6}$ (Tabela 2) podem resultar de restos de construção incorporados aos solos. Em faixas normais de $\mathrm{pH}$ dos solos, as espécies iônicas $\mathrm{Pb}^{2+}$ e $\mathrm{Pb}(\mathrm{OH})^{+}$predominam na solução, o que aumenta a lixiviação desse poluente. Apenas para valores de $\mathrm{pH}$ superiores a 8 - 9 a forma precipitada $\mathrm{Pb}(\mathrm{OH})_{2}$ torna-se importante (HARTER, 1983; AKBAR et al., 2006). Os coeficientes de correlação entre teores de Pb trocável e pH em água foram negativos e altamente significativos $(r=-0,8 ; \mathrm{p}<0,016)$.

Tabela 2 - Características químicas e teor de argila dos solos

(continua)

\begin{tabular}{|c|c|c|c|c|c|c|c|c|c|c|c|c|c|c|}
\hline Amostra & $\begin{array}{c}\mathrm{pH} \\
\mathrm{H}_{2} \mathrm{O}\end{array}$ & $\begin{array}{c}\mathrm{pH} \\
\mathrm{CaCl}_{2}\end{array}$ & $\mathbf{A l}^{3+}$ & $\mathbf{H}+\mathbf{A l}^{3+}$ & $\mathrm{Ca}^{2+}$ & $\mathbf{M g}^{2+}$ & $\mathbf{K}^{+}$ & $\mathrm{Na}^{+}$ & $\begin{array}{l}\text { CTC } \\
\text { total }\end{array}$ & $\mathrm{CO}^{(1)}$ & V & $\mathrm{m}$ & $\operatorname{Arg}^{(2)}$ & $\mathbf{T}^{(3)}$ \\
\hline & & & \multicolumn{7}{|c|}{ - $\mathrm{cmol}_{\mathrm{c}} \mathrm{kg}^{-1}$} & $\mathrm{~g} \mathrm{~kg}^{-1}$ & \multicolumn{2}{|c|}{ - $\%$} & $\mathrm{~g} \mathrm{~kg}^{-1}$ & $\mathrm{cmol}_{\mathrm{c}} \mathrm{kg}^{-1}$ \\
\hline $\mathrm{P}_{1} / \mathrm{A}$ & 4,7 & 3,9 & 4,1 & 42,7 & 4,7 & 3,2 & 0,45 & 0,19 & 51,2 & 105,0 & 16,7 & 32,7 & 291 & 176,1 \\
\hline $\mathrm{P}_{1} / \mathrm{C}$ & 5,0 & 3,9 & 2,5 & 18,0 & 3,4 & 2,6 & 0,07 & 0,03 & 24,1 & 23,2 & 25,3 & 28,9 & 203 & 118,7 \\
\hline $\mathrm{P}_{2}$ & 4,7 & 4,2 & 1,2 & 25,8 & 5,3 & 2,2 & 0,52 & 0,04 & 33,9 & 94,8 & 23,8 & 13,4 & 368 & 92,0 \\
\hline
\end{tabular}

Rev. Acad., Ciênc. Agrár. Ambient., Curitiba, v. 8, n. 1, p. 47-53, jan./mar. 2010 
Tabela 2 - Características químicas e teor de argila dos solos

\begin{tabular}{|c|c|c|c|c|c|c|c|c|c|c|c|c|c|c|}
\hline $\mathrm{P}_{3}$ & 4,9 & 4,3 & 0,2 & 9,4 & 12,2 & 4,5 & 0,48 & 0,13 & 26,7 & 60,6 & 64,8 & 1,1 & 158 & 169,1 \\
\hline $\mathrm{P}_{4}$ & 4,9 & 3,9 & 1,9 & 12,3 & 1,2 & 2,8 & 0,29 & 0,04 & 16,6 & 36,5 & 26,0 & 30,8 & 450 & 37,0 \\
\hline $\mathrm{P}_{5}$ & 6,5 & 5,7 & 0,1 & 3,1 & 8,5 & 4,5 & 0,37 & 0,05 & 16,5 & 37,7 & 81,2 & 0,7 & 160 & 103,3 \\
\hline $\mathrm{P}_{6}$ & 6,9 & 6,4 & 0,1 & 1,5 & 11,5 & 5,9 & 0,70 & 0,05 & 19,7 & 30,5 & 92,3 & 0,9 & 413 & 47,6 \\
\hline $\mathrm{P}_{7}$ & 5,3 & 4,0 & 3,4 & 20,6 & 0,9 & 2,0 & 0,20 & 0,03 & 23,7 & 40,2 & 13,2 & 52,4 & 550 & 43,1 \\
\hline $\mathrm{R}_{1} / \mathrm{A}$ & 5,8 & 4,1 & 2,5 & 25,6 & 1,7 & 2,2 & 0,12 & 0,16 & 29,8 & 59,4 & 14,0 & 37,5 & 604 & 49,3 \\
\hline $\mathrm{R}_{1} / \mathrm{C}$ & 5,1 & 4,1 & 4,0 & 33,3 & 0,1 & 0,5 & 0,02 & 0,04 & 34,0 & 94,8 & 1,9 & 84,9 & 66 & 514,5 \\
\hline $\mathrm{R}_{2} / \mathrm{A}$ & 4,3 & 4,0 & 3,9 & 19,6 & nd & 1,8 & 0,08 & 0,04 & 21,5 & 31,7 & 8,9 & 67,4 & 491 & 43,8 \\
\hline $\mathrm{R}_{2} / \mathrm{B}$ & 4,5 & 3,9 & 3,0 & 6,8 & nd & 1,5 & 0,03 & 0,02 & 8,4 & 5,1 & 18,5 & 66,3 & 467 & 17,9 \\
\hline $\mathrm{R}_{2} / \mathrm{C}$ & 4,7 & 3,9 & 3,6 & 5,8 & nd & 0,8 & 0,02 & 0,00 & 6,6 & 2,7 & 12,3 & 80,7 & 449 & 14,7 \\
\hline
\end{tabular}

(1) $\mathrm{CO}=$ carbono orgânico. ${ }^{(2)}$ Arg. $=$ Teor de argila. ${ }^{(3)} \mathrm{T}=$ Atividade da fração argila ou CTC da fração argila: CTC x 100 / \% argila (EMBRAPA, 2006). nd = valores abaixo do nível de detecção do método analítico (espectrometria por absorção atômica).

Como observado em outros estudos (AKBAR et al., 2006; DOLAN et al., 2006), os teores totais de $\mathrm{Pb}$ foram maiores nos solos próximos às rodovias (Tabela 3). A amostra $\mathrm{P}_{3}$ apresentou teor de $\mathrm{Pb}$ total cerca de dez vezes superior à amostra $\mathrm{R}_{2}$ tomada como referência (mesmo material de origem e unidade pedológica - Tabela 1). Esse local situava-se ao fim de uma rampa, logo após o pedágio (sentido Curitiba-Paranaguá), o que lhe confere maior possibilidade de lavagem e acúmulo de $\mathrm{Pb}$ depositado sobre a pista, em relação aos demais pontos sob as mesmas condições $\left(\mathrm{P}_{4}\right.$ e $\left.\mathrm{P}_{5}\right)$. A permanência de veículos com o motor em funcionamento em longas filas na região do pedágio deve contribuir para maior deposição de $\mathrm{Pb}$ particulado na rodovia.

O ponto $\mathrm{P}_{1}$ é o único que não recebe diretamente águas de escorrimento da rodovia, pois se situa em cota mais alta que o nível do pavimento (perfil em barranco à margem da rodovia). $\mathrm{O}$ incremento de $\mathrm{Pb}$ nos horizontes $\mathrm{A}$ e $\mathrm{Cg}$ desse perfil em relação à referência $\mathrm{R}_{1}$ (Tabela 3) pode ser atribuído à deposição direta do metal particulado presente na fumaça liberada pelos veículos. $\mathrm{O}$ ponto $\mathrm{P}_{2}$, de mesma feição pedológica, apresentou teores similares de $\mathrm{Pb}$ em relação ao $\mathrm{P}_{1}$.

Pires et al. (2007) determinaram os teores totais de $\mathrm{Pb}$ em diferentes solos originados de argilito da Formação Guabirotuba na região metropolitana de Curitiba. No horizonte A de um Cambissolo (mesma classe e material de origem dos pontos $\mathrm{P}_{6}$ e $\mathrm{P}_{7}$, na BR 116 - Tabela 1), o teor foi de 3,2 $\mathrm{mg} \mathrm{kg}^{-1}$, muito inferior aos observados nos pontos $\mathrm{P}_{6}$ e $\mathrm{P}_{7}\left(54,5\right.$ e 33,2 $\mathrm{mg} \mathrm{kg}^{-1}$, respectivamente) (Tabela 3), o que sugere contaminação decorrente do tráfego de veículos também na BR 116. Viard et al. (2004), em estudo dos efeitos de bioacumulação de $\mathrm{Pb}, \mathrm{Zn}$ e $\mathrm{Cd}$ em solos próximos a rodovias de tráfego intenso, concluíram que essa induz a uma contaminação sobre o ambiente circundante, até $320 \mathrm{~m}$, mas com o máximo de contaminação observados entre $5 \mathrm{~m}$ e $20 \mathrm{~m}$ da rodovia.

$\mathrm{O} \mathrm{Pb}$ na forma trocável (adsorvido eletrostaticamente) está presente em todos os solos amostrados (Tabela 3), sem uma distinção clara entre as amostras coletadas próximo às rodovias e aos pontos de referências. Como era previsto, o aumento na CTC total dos solos foi importante para adsorção de maior quantidade de $\mathrm{Pb}$ na forma trocável $(\mathrm{r}=0,85 ; \mathrm{p}<0,006)$.

A diferença considerável entre os teores totais e trocáveis de $\mathrm{Pb}$ (Tabela 3) indica sua baixa mobilidade no solo e evidencia que o elemento encontra-se nas amostras em formas químicas mais estáveis - por exemplo, formando complexos de esfera interna (KABATA-PENDIAS; PENDIAS, 2001; JI; LI, 1997; LI, 2006). 
Tabela 3 - Teores de Pb total e trocável nos solos

\begin{tabular}{crr}
\hline Amostra & Pb total & \multicolumn{2}{c}{ Pb trocável } \\
\cline { 2 - 3 } $\mathrm{P}_{1} / \mathrm{A}$ & 41,7 & \\
$\mathrm{P}_{1} / \mathrm{C}$ & 44,1 & 13,3 \\
$\mathrm{P}_{2}$ & 52,2 & 6,7 \\
$\mathrm{P}_{3}$ & 215,1 & 8,5 \\
$\mathrm{P}_{4}$ & 21,6 & 9,5 \\
$\mathrm{P}_{5}$ & 27,9 & 7,4 \\
$\mathrm{P}_{6}$ & 54,5 & 2,2 \\
$\mathrm{P}_{7}$ & 33,2 & 3,7 \\
$\mathrm{R}_{1} / \mathrm{A}$ & 19,3 & 5,1 \\
$\mathrm{R}_{1} / \mathrm{C}$ & 7,3 & 3,9 \\
$\mathrm{R}_{2} / \mathrm{A}$ & 22,4 & 6,8 \\
$\mathrm{R}_{2} / \mathrm{B}$ & 25,8 & 6,5 \\
$\mathrm{R}_{2} / \mathrm{C}$ & 11,5 & 2,1 \\
\hline
\end{tabular}

\section{Conclusões}

- Verificou-se incremento nos teores de $\mathrm{Pb}$ nos solos às margens dos trechos estudados das rodovias, em relação aos solos de referência, principalmente para as amostras coletadas em locais sujeitos à passagem de águas de escorrimento superficial da pista;

- A contaminação foi favorecida nos solos situados ao fim de longas rampas, pela maior possibilidade de captação de $\mathrm{Pb}$ particulado depositado sobre as rodovias pelos escapamentos dos veículos;

- Os teores de Pb trocável foram positivamente correlacionados com os valores de capacidade de troca de cátions (CTC) total dos solos coletados próximo às rodovias.

\section{Referências}

AKBAR, K. F. et al. Heavy metal contamination of roadside soils of Northern England. Soil and Water Research, v. 4, n. 1, p. 158-163, 2006.

AMARAL SOBRINHO, N. M. B. et al. Mobilidade de metais pesados em solo tratado com resíduo siderúrgico. Revista Brasileira de Ciência do Solo, v. 22, n. 2, p. 345-353, 1998.

ANDRADE, S. A. L. et al. Interação de chumbo, da saturação por bases do solo e de micorriza arbuscular no crescimento e nutrição mineral da soja. Revista Brasileira de Ciência do Solo, v. 27, n. 5, p. 955-959, 2003.

ANDRÉ, E. M. et al. Frações de zinco em solo arenoso e suas relações com disponibilidade para Cynodon spp. Revista Brasileira de Ciência do Solo, v. 27, n. 3, p. 451-459, 2003.

DOLAN, L. M. J. et al. Towards the sustainable development of modern road ecosystem. In: DAVENPORT, J.; DAVENPORT, J. L. (Ed.). The ecology of transportation: managing mobility for the environment. Netherlands: Springer, 2006. p. 275-331.

EMPRESA BRASILEIRA DE PESQUISA AGROPECUÁRIA - EMBRAPA. Manual de métodos de análise de solo. 2. ed. Rio de Janeiro: Centro Nacional de Pesquisa de Solos, 1997.

. Manual de análises químicas de solos, plantas e fertilizantes. Brasília: Embrapa Comunicação para Transferência de Tecnologia, 1999. 
Sistema brasileiro de classificação de solos. Rio de Janeiro: Centro Nacional de Pesquisa de Solos, 2006.

GOMES, P. C. et al. Extração fracionada de metais pesados em Latossolo Vermelho Amarelo. Revista Brasileira de Ciência do Solo, v. 21, n. 3, p. 543-551, 1997.

HARTER, R. D. Effect of soil pH on adsorption of lead, copper, zinc, and nickel. Soil Science Society of America Journal, v. 47, n. 1, p. 47-51, 1983.

JI, G. L.; LI, H. Y. Electrostatic adsorption of cations. In: YU, T. R. Chemistry of variable charge soils. New York: Oxford University Press, 1997. p. 65-79.

KABATA-PENDIAS, A.; PENDIAS, H. Trace elements in soils and plants. 3rd ed. Boca Raton: CRC Press, 2001.

LANDRIGAN, P. J. The worldwide problem of lead in petrol. Bulletin of World Health Organization, v. 80, n. 10 , p. $768,2002$.

LI, L. Y. Retention capacity and environmental mobility of $\mathrm{Pb}$ in soils along highway corridor. Water, Air, and Soil Pollution, v. 170, n. 1/4, p. 211-277, 2006.

LIM, C. H.; JACKSON, M. L. Dissolution for total elemental analysis. In: PAGE, A. L. Methods of soil analysis. Part 2: chemical and microbiological properties. Madison: American Society of Agronomy, 1986. p. 1-12.

NORRSTRÖM, A. C.; JACKS, G. Concentration and fractionation of heavy metals in roadside soils receiving de-icing salts. The Science of the Total Environment, v. 218, n. 2/3, p. 161-174, 1998.

PIRES, A. C. D. et al. Majors soil classes of the metropolitan region of Curitiba (PR), Brazil: II - interaction of Pb with mineral and organic constituents. Brazilian Archives of Biology and Technology, v. 50, n. 2, p. 183-192, 2007.

SUTHERLAND, R. A. et al. Characterization of selected element concentrations and enrichment ratios in background and anthropogenically impacted roadside areas. Archives of Environmental Contamination and Toxicology, n. 38, p. 428-438, 2000.

TURER, D.; MAYNARD, J. B. Heavy metal contamination in highway soils. Comparison of Corpus Christi, TX and Cincinnati, OH shows organic matter is key to mobility. Clean Technology and Environmental Policy, v. 4, p. 235-245, 2003.

VANZ, A.; MIRLEAN, N.; BAISCH, P. Avaliação de poluição do ar por chumbo particulado: uma abordagem geoquímica. Química Nova, v. 26, n. 1, p. 25-29, 2003.

VIARD, B. et al. Integrated assessment of heavy metal ( $\mathrm{Pb}, \mathrm{Zn}, \mathrm{Cd})$ highway pollution: bioaccumulation in soil, graminaceae and land snails. Chemosphere, v. 55, n. 10, p.1349-1359, 2004.

WENG, L. et al. Complexation with dissolved organic matter and solubility control of heavy metals in a sandy. Soil Environmental Science Technology, v. 36, n. 22, p. 4804-4810, 2002.

WORLD HEALTH ORGANIZATION - WHO. Enviromental Health Criteria 165: Inorganic Lead. International Programme on Chemical Safety. Geneva: World Health Organization, 1995.

WU, Z. et al. Effects of organic acids on adsorption of lead onto montmorillonite, goethite and humic acid. Environmental Pollution, v. 121, n. 3, p. 469-475, 2003.

Recebido: 16/06/2009

Received: 06/16/2009

Aprovado: 22/12/2009

Approved: 12/22/2009 